\title{
Cada vez más evidencia en contra de la realización de rastreo de cáncer prostático en la población general
}

\author{
More evidence against prostatic cancer screening in the general population
}

Karin Kopitowski , Ignacio Vicente Lago , Valeria Vietto , Marcela Botargues y Sergio Terrasa

\begin{abstract}
Resumen
La Fuerza de Tareas de Cuidados Preventivos de EE.UU. expidió su recomendación final en contra de la realización de rastreo de cáncer de próstata a través de la determinación sérica de antígeno prostático específico en la población general asintomática (recomendación tipo D), por considerar que existe moderada o alta certeza de que este servicio no tiene beneficio neto. Este artículo describe la principal evidencia que sustenta esta recomendación.
\end{abstract}

\begin{abstract}
The U.S. Preventive Services Task Force issued its final recommendation against the implementation of prostate cancer screening through the determination of serum prostate-specific antigen in the general asymptomatic population (type $\mathrm{D}$ recommendation), on the grounds that there is moderate to high certainty that this service has no net benefit. This article describes the most important evidence that support this recommendation.
\end{abstract}

Palabras clave: antígeno prostático específico, cáncer prostático, rastreo. Key words: prostatic specific antigen, prostatic cancer, screening.

Kopitowski K, Vicente Lago I, Vietto V y Terrasa S. Cada vez más evidencia en contra de la realización de rastreo de cáncer prostático en la población general. Evid Act Pract Ambul. 15(2).59-63 Abr-Jun 2012.

Introducción

El 21 de mayo de 2012, la Fuerza de Tareas de Cuidados Preventivos de EE.UU. (en inglés: U.S. Preventive Task Force o USPSTF) expidió su recomendación final en contra de la realización de rastreo de cáncer de próstata a través de la determinación sérica de Antígeno Prostático Específico (en inglés prostatic specific antigen: PSA) en la población general asintomática ${ }^{1}$ (recomendación tipo D), por considerar que existe moderada o alta certeza de que esta prueba no tiene beneficio neto o que los daños superan a los beneficios que provoca. En este artículo se resumen los resultados de las revisiones sistemáticas llevadas a cabo por dicha institución con el propósito de sintetizar la evidencia actual sobre rastreo y tratamiento del cáncer de próstata localizado, sobre los que se fundamenta esta recomendación ${ }^{2}$.

A fines de ordenar la discusión, los resultados de los estudios primarios disponibles fueron revisados y analizados en un marco estructurado en las siguientes cuatro preguntas:

\section{1) ¿El rastreo con antígeno prostático específico disminuye la mortalidad por cáncer de próstata o la mortalidad por todas las causas?}

Con el objetivo primario de comparar la mortalidad por cáncer de próstata $(\mathrm{CP})$ en pacientes asignados aleatoriamente a rastreo de esta patología con determinación de PSA versus el cuidado usual instituido por su equipo tratante, el Estudio Europeo Aleatorizado de Rastreo de Cáncer de Próstata ${ }^{3+\dagger}$ (ERSPC por sus siglas en inglés) documentó una mayor incidencia de $\mathrm{CP}$ en el grupo de pacientes asignado a rastreo (con un aumento neto de 34 casos por mil pacientes rastreados), pero no logró detectar diferencias estadísticamente significativas en la mortalidad general por esta enfermedad (RR: 0,85; IC95\% 0,73 a 1). Sin embargo, en el subgrupo de personas de 55 a 69 años el rastreo se asoció a un $20 \%$ de reducción de la mortalidad (RR: 0,8; IC95\% 0,6 a 0,98), con una reducción del riesgo absoluto (RRA) de $0,07 \%$ y la necesidad de rastrear 1410 hombres y tratar 48 para prevenir una muerte por $\mathrm{CP}^{\neq \neq}$. Por otro lado, un estudio realizado en Göteborg, Suecia ${ }^{4 \S \S}$ (centro incluido en el ERSPC ${ }^{4}$ y cuyos resultados fueron publicados de forma independiente) también reportó un incremento de la incidencia de cáncer de próstata (HR 1,6; IC95\% 1,5 a 1,8), y describió una reducción estadísticamente significativa de la mortalidad específica por esta causa (RR 0,56; IC95\% 0,39 a $0,82)$, con una RAA de $0,4 \%(0,17$ a $0,64 \%)$, estimándose que haría falta rastrear 293 personas (IC95\% 177 a 799) y tratar a 12 para prevenir una muerte por esta enfermedad ${ }^{\star \star *}$.

Por su parte, el ensayo clínico aleatorizado de rastreo de cáncer prostático, pulmonar, colorrectal y de ovario ${ }^{5}$ (PLCO por sus siglas en inglés) comparó los resultados de una estrategia de rastreo combinada (dosaje de PSA más tacto rectal anual) con los cuidados usuales ${ }^{t+t}$. Se observó un $20 \%$ de aumento en la incidencia de cáncer de próstata (RR 1,2; IC95\% 1,1 a $1,3)$ sin reducción significativa de la mortalidad específica (RR $1,13 ; 0,75$ a 1,70$)$ ni de la mortalidad global (RR 0,97; 0,93 a $1,01)$ a los diez años de seguimiento ${ }^{\ddagger \pm}$.

Otros tres ensayos clínicos de pobre calidad metodológica ${ }^{\S \varsigma}$ (dos ${ }^{6,7}$, de los cuales habían sido incluidos en la revisión de la USPSTF de $2008^{8}$ ) no encontraron diferencias significativas en la mortalidad específica por CP entre los participantes invitados a realizar rastreo y los asignados a los grupos control ${ }^{7,9,10}$.

"Ensayo clínico multicéntrico que evaluó un total de 182.000 pacientes provenientes de siete países de la Unión Europea, con un rango etario de 50 a 74 años y una mediana de seguimiento de nueve años.

${ }^{\ddagger}$ Este estudio, considerado por la Fuerza de Tareas de Preventiva de EE.UU. (USPSTF) como de regular calidad, tuvo varias limitaciones metodológicas: fueron muy variables entre los diferentes centros el intervalo de medición del PSA (rango de 2 a 7 años) y el punto de corte para el diagnóstico (rango entre 2,5 a 4 ng/dL, aunque uno de los centros empleó $10 \mathrm{ng} / \mathrm{dL}$ por varios años); se registraron diferencias en la edad de elegibilidad y en los procesos de reclutamiento y aleatorización, fueron excluidos dos centros por razones no reportadas y tampoco fue reportada la pérdida en el seguimiento.

\$s Fueron evaluados 19.904 pacientes de 50 a 64 años, de los cuales 9952 recibieron invitación a realizar rastreo con PSA cada dos años (punto de corte 2,5 - 3 ), y $76 \%$ de los cuales tuvo como mínimo una medición, con una mediana de seguimiento reportada de 14 años.

los cuales tuvo como mínimo una medición, con una mediana de seguimiento reportada de 14 años. ${ }^{* * *}$ Como limitaciones de este estudio, considerado por la USPSTF como de regular calidad, se destacan la ausencia se información sociodemográfica de ambos grupo
contaminación de los controles que se estima en un $3 \%$ aunque no fue evaluada, y la inclusión de los resultados del $60 \%$ de los pacientes en el análisis del ERSPC.

ttt Estudio multicéntrico que evaluó un total de 76.693 pacientes, en un rango etario de 55 a 74 años, con una media de seguimiento reportada de 11,5 años. Se reportó una adherencia del grupo intervenido de $85 \%$ para PSA (punto de corte $4 \mathrm{ng} / \mathrm{dL}$ ) y $86 \%$ para tacto rectal.

\#\#te estudio fue considerado por la USPSTF como de regular calidad, destacándose como principal limitación una contaminación de los controles de 52\% (pacientes asignados al grupo control que tuvieron al menos una determinación de PSA durante el estudio). Además, $44 \%$ de los pacientes de cada grupo tenía al menos un dosaje de PSA antes de su ingreso al estudio.

${ }_{\$ \S \S}$ Las limitaciones metodológicas presentes en estos estudios incluyeron una inadecuada descripción de los métodos de aleatorización y ocultamiento de la asignación, así como de las pérdidas de seguimiento y una falta de claridad en el enmascaramiento de los evaluadores de los resultados. Uno de los estudios empleó un punto de corte inusualmente elevado de $\mathrm{PSA}^{10}$.

Servicio de Medicina Familiar y Comunitaria del Hospital Italiano de Buenos Aires. Departamento de Medicina del Instituto Universitario Hospital Italiano de Buenos Aires. karin.kopitowski@ hospitalitaliano.org.ar

tServicio de Medicina Familiar y Comunitaria del Hospital Italiano de Buenos Aires. ignacio.vicente@ hospitalitaliano.org.ar

Servicio de Medicina Familiar y Comunitaria del Hospital Italiano de Buenos Aires. valeria.vietto @ hospitalitaliano.org.ar

§ Servicio de Medicina Familiar y Comunitaria del Hospital Italiano de Buenos Aires. maria.botargues@ hiba.org.ar

** Servicio de Medicina Familiar y Comunitaria de Hospital Italiano de Buenos Aires. Departamento de Salud Pública de Instituto Universitario Hospital Italiano de Buenos Aires. sergio.terrasa@ hiba.org.ar 
2) ¿Cuáles son los daños que provoca el rastreo de cáncer de próstata con PSA?

Dos estudios reportaron los daños directos del rastreo de cáncer de próstata a través de determinación de PSA. Se observó que $76 \%$ de todas las biopsias prostáticas realizadas en el contexto del estudio ERSPC a causa de una elevación del PSA elevado fueron negativas para cáncer de próstata ${ }^{4}$, mientras que uno de los centros de este estudio reportó en forma independiente que $12 \%$ de sus participantes tenían por lo menos un resultado falsamente positivo luego de tres series de $\mathrm{PSA}^{11 * * * *}$. Por su parte, el reporte del estudio PLCO describió que cada paciente tiene un riesgo acumulativo de $13 \%$ de presentar al menos un resultado falso positivo luego de ser sometido a cuatro series de $\mathrm{PSA}^{\star \star * *}$, y una probabilidad de $5,5 \%$ de realizarse una biopsia como consecuencia de dicho resultado ${ }^{12}$

Las consecuencias físicas del rastreo en el estudio PLCO incluyeron sangrado intestinal o dolor anal (0,3 eventos por 10.000 participantes sometidos a tacto rectal), desmayo (26 eventos por cada 10.000 pacientes sometidos a una extracción de sangre), y complicaciones por la biopsia, como infección, sangrado o dificultades urinarias (68 eventos por cada 10.000 pacientes biopsiados) ${ }^{6}$. Para otro centro del ERSPC ${ }^{4}$ que publicó sus resultados en forma independiente, las complicaciones de la biopsia prostática transrectal más frecuentes fueron fiebre $(3,5 \%)$, retención aguda de orina $(0,4 \%)$ y hospitalización por urosepsis o prostatitis $(0,5 \%)^{13}$. Ningún estudio proporcionó información sobre potenciales daños psicológicos como consecuencia del rastreo.

\section{3) ¿Cuáles son los beneficios del tratamiento de cáncer de próstata en estadio temprano o detectado a través de una estrategia de rastreo?}

Con el objetivo de evaluar la mortalidad global y específica por CP en pacientes sometidos a prostatectomía en comparación con los sometidos a una conducta expectante, un ensayo clínico controlado aleatorizado ${ }^{14,15,16,17}$ de buena calidad metodológica evaluó 695 pacientes con cáncer de próstata localizadotttt, cuyos datos de seguimiento a lo largo de 15 años mostraron un descenso sostenido en el riesgo de morir por cáncer de próstata (RR 0,62; IC95\% 0,44 a 0,87) y una RRA de $6,1 \%$, lo que implica la necesidad de realizar (NNT)16 prostatectomías para evitar una muerte por esta enfermedad. Se observó además una reducción de la mortalidad global (RR 0,75; IC95\% 0,61 a 0,92) \#\#+. Además, se encontraron ocho estudios de cohorte ${ }^{\S \S \S}$ que mostraron consistentemente que la prostatectomía redujo la mortalidad global $^{18,19,20,21,22,23}$ (HR 0,46; rango 0,32 a 0,67) y la mortalidad por $\mathrm{CP}^{23,22,23,24,25}$ (HR 0,32; rango 0,25 a 0,50) en comparación con la conducta expectante. No se encontraron ensayos clínicos controlados aleatorizados que hubieran comparado el tratamiento radioterápico con la conducta expectante, pero sí cinco estudios de cohorte ${ }^{\star * \star *}$ que mostraron que la radioterapia disminuye mortalidad global (HR 0,68; rango 0,62 a 0,81) 22,22,26,24,26 y la mortalidad específica (HR 0,66; rango 0,63 a 0,70$)^{26,24,25,26}$ del

\section{CP localizado.}

\section{4) ¿Cuáles son los daños del tratamiento del cáncer de próstata en estadío temprano o detectado a través de una estrategia de rastreo?}

Se encontró sólo un ensayo clínico aleatorizado y controlado ${ }^{26}$ que había reportado los efectos adversos de la prostatectomía comparados con los asociados a mantener una conducta expectante en pacientes con estadios tempranos de CP detectado por rastreo. Se observó un mayor riesgo de incontinencia urinaria (RR 2,3; IC95\%1,6 a 3,2) y disfunción eréctil $(1,8$; IC95\% 1,5 a 2,2$)$ en el grupo intervenido, hallazgos consistentes con los reportados por estudios de cohorte cuyos RR promedio fueron 4,0 (rango 2,0 a 11) para incontinencia urinaria $^{27,28,29,30}$ y 1,5 (rango 1,3 a 2,1) para disfunción eréctil 29,30,31. El incremento del riesgo absoluto atribuido por el ensayo clínico a la prostatectomía fue de $28 \%$ en el caso de la incontinencia urinaria $(49 \%$ versus $21 \%)$, mientras que para disfunción eréctil fue de $36 \%(81 \% \text { versus } 45 \%)^{27}$.

Por otro lado, ocho estudios ${ }^{30,31,32,33,34,35,36,37}$ evaluaron el impacto de la prostatectomía sobre el autorreporte de calidad de vida a través del cuestionario SF-36 ${ }^{t+t+1+38}$. Dos de ellos ${ }^{31,33}$ reportaron puntajes globales muy similares en los componentes físico y mental, mientras que otros estudios que comunicaron subescalas específicas del SF-36, evidenciaron mejores puntajes en la funcionalidad física (diferencia de ocho puntos, con un rango de 2 a 16) ${ }^{30,33,34,35,37,38}$ y en la esfera emocional (diferencia de 8 puntos, rango -5 a 13) ${ }^{30,33,34,35,36,37,38}$ en el grupo que recibió tratamiento quirúrgico.

Un estudio poblacional de complicaciones a corto plazo de la prostatectomía ${ }^{39 \$ \$ s \$}$ describió una tasa de mortalidad perioperatoria de $0,5 \%$, similar a la reportada por otros estudios observacionales. La misma publicación informó una tasa de eventos cardiovasculares serios ${ }^{\star \star \star * \star *}$ de $3 \%$, y de otros eventos vasculares $^{t+t t t+}$ de $2 \%{ }^{39}$. Se encontró un único ensayo clínico aleatorizado que reportó niveles de ansiedad, depresión y preocupación en pacientes sometidos a prostatectomía en comparación con conducta expectante, que luego de cuatro años de seguimiento no encontró diferencias significativas entre ambos grupos $^{40}$. Con respecto al tratamiento radioterápico del CP temprano detectado por rastreo, se observó un mayor riesgo de incontinencia urinaria en comparación con la conducta expectante en un pequeño ensayo clínico aleatorizado y controlado, pero con una estimación del efecto muy imprecisa (RR 8,3; IC95\% 1,1 a 63) debido al escaso número de eventos ${ }^{41 \neq t \neq t \neq 1}$ Vale destacar que esta asociación no había quedado clara tampoco en los estudios de cohorte (RR promedio 1,1 ; rango $0,71$ a 2,0$)^{28,29,30,31}$. No se encontraron ensayos clínicos que hubieran evaluado el riesgo de disfunción eréctil asociado a radioterapia, pero seis estudios de cohorte ${ }^{31,32,30,31,32}$, reportaron un incremento de esta secuela en comparación con la conducta expectante (RR 1,3; rango 1,1 a 1,5).

\footnotetext{
Empleando un punto de corte de $4 \mathrm{ng} / \mathrm{dL}$.
}

t+t La inclusión de pacientes con cáncer de próstata localizado no estuvo limitada a aquellos detectados por estrategias de rastreo. $75 \%$ de los pacientes evaluados tenían tumores palpables (estadío T2), mientras que se encontraban en este estadío solamente $36 \%$ de los cánceres del estudio ERSPC.

\#\# el análisis de subgupos, el beneficio se restringió a hombres menores de 65 años de edad para mortalidad por cáncer de próstata (RR 0,49; IC95\% 0,37 a 0,79) y para mortalidad global (RR 0,52; IC95\% 0,37 a 0,73 ).

mortalidad global (RR 0,52; IC95\% 0,37 a 0,73).

\$s\$§ Estudios con un promedio de 2264 participantes (rango 316 a 25.900) con cáncer de próstata localizado y un rango de duración del seguimiento de cuatro a 13 años. ${ }^{\star \star \star \star \star \star}$ Estudios con un tamaño muestral promedio de 3441 pacientes (rango 334 a 30.000 ) y una duración de seguimiento de 4 a 13 años.

"ttrt Una diferencia de 6 a 9 puntos en la escala de SF-36 es considerada un beneficio clínico importante de efecto moderado en la calidad de vida.

\#\#\# El estudio de Bacon y col. reportó un puntaje global del componente físico de 2, mientras que el de Smith y col. fue de 3 . El puntaje global reportado para el componente mental fue de 0 y 3 respectivamente para cada estudio.

${ }^{\$ \varsigma \$ \varsigma \S}$ Este estudio descriptivo incluyó datos de un total de 101604 pacientes sometidos a prostatectomía obtenidos a partir de reclamos a Medicare, el programa nacional de seguro social de los Estados Unidos, definiendo como período de seguimiento aquel comprendido dentro de los 30 días posteriores a la intervención.

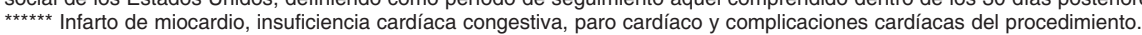

tIttt Tromboembolismo pulmonar o infarto pulmonar, tromboflebitis de vasos profundos de los miembros inferiores, embolismo o flebitis no especificada, trombosis o embolismo arterial y accidente cerebrovascular.

arterial y accidente cerebrovascular.

\#\#\#‡Este estudio incluyó 108 pacientes con CP localizado seleccionados aleatoriamente y un grupo control de 68 pacientes apareados por edad, reportándose sólo un caso de incontinencia urinaria en el grupo asignado a conducta expectante. 
Se evaluó el impacto de la radioterapia en el autorreporte de la calidad de vida a través del formulario SF-36 en diez estudios $^{33,34,33,37,38,36,36,37,42,43}$. Tres de ellos ${ }^{30,32,42}$ no detectaron diferencias significativas en los puntajes globales de los componentes físico y mental| ${ }^{\$ \$ s \S s}$, mientras que siete estudios que reportaron subescalas específicas del SF-36 evidenciaron mejores puntajes en funcionalidad física (diferencia de -9 puntos, rango -22 a $1)^{29,32,37,38,36,37,42}$ en el grupo asignado a conducta expectante.
Seis estudios de cohorte $33,30,32,34,36,42$ documentaron que la radioterapia se asoció a peores resultados que la conducta expectante $^{\star \star \star \star * \star * \star}$ en la funcionalidad intestinal, con mayor incidencia de efectos adversos intestinales durante los primeros meses posteriores a la radioterapia y una mejoría gradual luego de la misma ${ }^{37,36,43,44}$

La tabla 1 resume la principal evidencia disponible sobre la efectividad del rastreo de cáncer prostático.

Tabla 1: principal evidencia sobre la efectividad del rastreo de cáncer prostático.

\begin{tabular}{|c|c|c|c|c|c|}
\hline & $\begin{array}{c}\text { Estudios (n), y } \\
\text { calidad en general }\end{array}$ & Limitaciones & Consistencia & $\begin{array}{l}\text { Aplicabilidad a la } \\
\text { población de rastreo }\end{array}$ & Resumen de los hallazgos \\
\hline $\begin{array}{l}\text { ¿El rastreo con PSA } \\
\text { disminuye la mortalidad } \\
\text { por cáncer de próstata o } \\
\text { la mortalidad por todas } \\
\text { las causas? }\end{array}$ & $\begin{array}{l}5 \text { ECCA } \\
\text { Calidad en } \\
\text { general: buena }\end{array}$ & \begin{tabular}{|l|} 
Sólo 2 ECCA de buena calidad, \\
un reporte adicional de buena \\
calidad de un centro \\
participante de uno de los ECCA \\
con solapamiento de pacientes.
\end{tabular} & $\begin{array}{l}\text { Baja (resultados } \\
\text { discordantes entre los } \\
\text { ECCA de más alta } \\
\text { calidad) }\end{array}$ & $\begin{array}{l}\text { Algunas prácticas de } \\
\text { rastreo (intervalo y } \\
\text { punto de corte de PSA) } \\
\text { fueron diferentes de la } \\
\text { práctica típica en } \\
\text { EE.UU. }\end{array}$ & $\begin{array}{l}\text { El rastreo con PSA identifica casos adicionales } \\
\text { de CP, pero la mayoría de los ensayos clínicos } \\
\text { no ha demostrado una reducción } \\
\text { estadísticamente significativa de la mortalidad } \\
\text { específica por CP. } \\
\text { El ensayo ERSPC encontró que el rastreo con } \\
\text { PSA cada } 2 \text { a } 7 \text { años, en un subgrupo de } \\
\text { individuos de } 55 \text { a } 69 \text { años y luego de } 9 \text { años de } \\
\text { seguimiento, se asoció a un } 20 \% \text { de reducción } \\
\text { de la mortalidad (RR: } 0,8 ; \text { IC95\% } 0,6 \text { a } 0,98 \text { ), } \\
\text { RRA 0,07\%; sin embargo el ensayo PLC0 no } \\
\text { encontró efecto luego de } 10 \text { años de } \\
\text { seguimiento (RR } 1,13 ; \text { IC95\% } 0,75 \text { a } 1,70 \text { ). } \\
\text { Un estudio de buena calidad de uno de los } \\
\text { centros incluido en el ERSPC reportó mejores } \\
\text { resultados que los del análisis general del } \\
\text { ERSPC, con solapamiento sustancial de } \\
\text { pacientes. Tres estudios de baja calidad no } \\
\text { encontraron diferencias significativas. }\end{array}$ \\
\hline $\begin{array}{l}\text { ¿Cuáles son los daños } \\
\text { que provoca el rastreo } \\
\text { de CP con PSA? }\end{array}$ & $\begin{array}{l}2 \text { ECCA } \\
\text { Calidad en } \\
\text { general: buena }\end{array}$ & $\begin{array}{l}\text { Evidencia aleatorizada } \\
\text { disponible sólo de } 2 \text { ECCA de } \\
\text { buena calidad. }\end{array}$ & Alta & $\begin{array}{l}\text { Algunas prácticas de } \\
\text { rastreo (intervalo y } \\
\text { punto de corte de PSA) } \\
\text { fueron diferentes de la } \\
\text { práctica típica en } \\
\text { EE.UU. }\end{array}$ & $\begin{array}{l}\text { Dos estudios reportaron tasas de falsos } \\
\text { positivos entre } 12 \text { y } 13 \% \text { luego de } 3-4 \text { series de } \\
\text { rastreo con PSA; uno de ellos informó que } 76 \% \\
\text { de las biopsias de próstata no identificaron } \\
\text { cáncer. Infecciones o retención urinarias en } 0.5 \\
\text { a } 1 \% \text { de las biopsias. Evidencia insuficiente para } \\
\text { estimar la magnitud de los daños psicológicos } \\
\text { asociados a falsos positivos del PSA. }\end{array}$ \\
\hline \multirow[t]{2}{*}{$\begin{array}{l}\text { ¿Cuáles son los } \\
\text { beneficios del } \\
\text { tratamiento de CP en } \\
\text { estadio temprano o } \\
\text { detectado a través de } \\
\text { una estrategia de } \\
\text { rastreo? }\end{array}$} & $\begin{array}{l}\text { Prostatectomía } \\
9 \text { estudios: } \\
1 \text { ECCA, } \\
8 \text { estudios de } \\
\text { cohorte } \\
\text { Calidad en } \\
\text { general: buena }\end{array}$ & Sólo un ECCA & Alta & $\begin{array}{l}\text { Los casos de CP en el } \\
\text { ECCA fueron } \\
\text { detectados por sus } \\
\text { manifestaciones } \\
\text { clínicas más que por } \\
\text { rastreo, y había una } \\
\text { alta proporción de CP } \\
\text { en estadios T2. } \\
\text { Limitada información } \\
\text { sobre las técnicas } \\
\text { quirúrgicas evaluadas. }\end{array}$ & $\begin{array}{l}\text { La prostatectomía se asoció a descenso en el } \\
\text { riesgo de mortalidad por CP (RR 0,62; IC } 95 \% \\
0,44 \text { a 0,87) y de mortalidad global (RR 0,75; } \\
\text { IC95\% 0,61 a 0,92), RRA 6,6\% (IC95\% -1,3 a } \\
14 \% \text { ) en comparación con la conducta } \\
\text { expectante luego de } 15 \text { años de seguimiento en } \\
\text { un ECCA de buena calidad. Análisis de } \\
\text { subgrupos sugieren que el benficicio estaría } \\
\text { limitado a pacientes < } 65 \text { años de edad. } \\
\text { Estudios observacionales también mostraron } \\
\text { que la prostatectomía se asoció a reducción de } \\
\text { la mortalidad global (HR 0,46; rango 0,32 a } \\
0,67) \text { y específica por CP (HR 0,32; rango 0,25 a } \\
0,50) \text { luego de } 4 \text { a } 13 \text { años de seguimiento } \\
\text { cuando fue comparada con conducta } \\
\text { expectante. }\end{array}$ \\
\hline & $\begin{array}{l}\text { Radioterapia } \\
5 \text { estudios de } \\
\text { cohorte } \\
\text { Calidad en } \\
\text { general: buena }\end{array}$ & No se encontraron ECCA. & Alta & $\begin{array}{l}\text { Información limitada } \\
\text { sobre las técnicas de } \\
\text { radioterapia } \\
\text { específicas y los } \\
\text { regímenes evaluados. }\end{array}$ & $\begin{array}{l}\text { La radioterapia disminuye la mortalidad global } \\
\text { (HR 0,68; rango } 0,62 \text { a } 0,81 \text { ) y específica (HR } \\
0,66 ; \text { rango } 0,63 \text { a } 0,70 \text { ) del CP localizado, } \\
\text { luego de } 4 \text { a } 13 \text { años de seguimiento y en } \\
\text { comparación con conducta expectante. }\end{array}$ \\
\hline
\end{tabular}




\begin{tabular}{|c|c|c|c|c|c|}
\hline \multirow[t]{2}{*}{$\begin{array}{l}\text { ¿Cuáles son los daños } \\
\text { del tratamiento de CP en } \\
\text { estadio temprano o } \\
\text { detectado a través de } \\
\text { una estrategia de } \\
\text { rastreo? }\end{array}$} & $\begin{array}{l}\text { Prostatectomía } \\
18 \text { estudios: } \\
1 \text { ECCA, } \\
11 \text { estudios de } \\
\text { cohorte, } \\
6 \text { estudios } \\
\text { observacionales } \\
\text { no controlados } \\
\text { Calidad en } \\
\text { general: buena }\end{array}$ & $\begin{array}{l}\text { Sólo un ECCA de buena calidad. } \\
\text { Estimaciones de riesgo no } \\
\text { ajustadas para incontinencia } \\
\text { urinaria y disfunción eréctil en } \\
\text { los estudios de cohorte. } \\
\text { Sólo un ECCA. }\end{array}$ & Moderada & $\begin{array}{l}\text { Limitada información } \\
\text { sobre las técnicas } \\
\text { quirúrgicas específicas } \\
\text { evaluadas. }\end{array}$ & $\begin{array}{l}\text { La prostatectomía se asoció a un mayor riesgo } \\
\text { de incontinencia urinaria (RR } 2,3 ; \text { IC95\% } 1,6 \text { a } \\
3,2 \text { ) y disfunción eréctil (1,8; IC95\% 1,5 a 2,2) } \\
\text { en un ECCA que comparó este tratamiento con } \\
\text { conducta expectante. En los estudios de cohorte } \\
\text { los RR promedio observados fueron 4,0 (rango } \\
2,0 \text { a 11) para incontinencia urinaria y de 1,5 } \\
\text { (rango 1,3 a 2,1) para disfunción eréctil. } \\
\text { En estudios poblacionales, la prostatectomía se } \\
\text { asoció con un riesgo de mortalidad } \\
\text { perioperatoria de } 0,5 \% \text { y de eventos } \\
\text { cardiovasculares serios de } 3 \% \text {. No se } \\
\text { observaron diferencias en el puntaje global del } \\
\text { SF-36 y la mayoría de sus subescalas. } \\
\end{array}$ \\
\hline & $\begin{array}{l}\text { Radioterapia } \\
14 \text { estudios: } 1 \\
\text { ECCA, } 13 \text { estudios } \\
\text { de cohorte } \\
\text { Calidad en } \\
\text { general: buena }\end{array}$ & $\begin{array}{l}\text { Estimaciones de riesgo no } \\
\text { ajustadas para incontinencia } \\
\text { urinaria y disfunción eréctil en } \\
\text { los estudios de cohorte }\end{array}$ & Moderada & $\begin{array}{l}\text { Información limitada } \\
\text { sobre las técnicas de } \\
\text { radioterapia } \\
\text { específicas y los } \\
\text { regímenes evaluados. }\end{array}$ & $\begin{array}{l}\text { La radioterapia se asoció a mayor riesgo de } \\
\text { incontinencia urinaria en comparación con la } \\
\text { conducta expectante en un pequeño ECCA con } \\
\text { una estimación del efecto muy imprecisa (RR } \\
8,3 \text {; IC95\% } 1,1 \text { a } 63 \text { ), pero no en los estudios de } \\
\text { cohorte (RR promedio 1,1; rango 0,71 a 2,0). } \\
\text { Además se asoció a un incremento del riesgo } \\
\text { de disfunción eréctil en comparación con } \\
\text { conducta expectante en estudios de cohorte (RR } \\
1,3 ; \text { rango } 1,1 \text { a 1,5), y de disfunción intestinal, } \\
\text { que parece mejorar con el tiempo. } \\
\text { No se observaron diferencias en el puntaje } \\
\text { global del SF-36 y la mayoría de sus } \\
\text { subescalas. }\end{array}$ \\
\hline
\end{tabular}

\section{Comentario}

Si bien la estrategia de rastreo de CP con PSA permite detectar casos adicionales de CP (el 90\% localizados), la mayoría de los ensayos clínicos no ha podido demostrar una reducción estadísticamente significativa de la mortalidad específica por CP. Por ejemplo, y como describimos previamente, los dos ensayos más grandes y de mejor calidad (ERSPC y PLCO) reportaron resultados contradictorios. El ERSPC reportó que el rastreo con PSA cada dos a siete años entre los 55 y los 69 años de edad se asocia con un $20 \%$ de reducción relativa del riesgo de morir por CP; mientras que el PLCO no encontró diferencias con la no realización de rastreo.

Vale destacar que la población que intervino en el PLCO había sido sometida previamente a altas tasas de rastreo de $\mathrm{CP}$, lo que podría haber atenuado las diferencias entre los grupos asignados a las diferentes intervenciones en el ensayo clínico. Sin embargo, si bien este "nivel de contaminación de los grupos" podría haber reducido la capacidad para documentar diferencias, no explica la tendencia observada de una mayor mortalidad específica por CP en el grupo intervenido ${ }^{t+t+t t t}$.

El rastreo con PSA se asocia con daños potenciales, incluyendo los relacionados a la evaluación subsiguiente de resultados falsos positivos, pero también al sobrediagnóstico de pacientes con formas CP de buena evolución que no se asociarían a un aumento de la morbimortalidad. Esto implica que estos pacientes quedarán expuestos a los daños innecesarios del consecuente sobretratamiento sin obtener un beneficio en términos de reducción de su morbimortalidad. Según los datos del ERSPC, la tasa de sobrediagnóstico se estima en el $50 \%$, y se evitaría la muerte por CP de un hombre por cada 48 pacientes sometidos a tratamiento. Mirando esta problemática desde los "efectos adversos del tratamiento", cada tres hombres sometidos a prostatectomía o cada siete sometidos a radioterapia en lugar de conducta expectante, se observaría un caso adicional de disfunción eréctil; mientras que cada cinco hombres tratados con prostatectomía se observaría un caso adicional de incontinencia urinaria.

Aún si la estrategia de rastreo poblacional en individuos asintomáticos fuera efectiva -lo que cada vez está más lejos de demostrarse por lo menos en la población general, ya que los resultados de los principales trabajos marcan la tendencia opuesta- todavía quedaría por definir su mejor intervalo de aplicación, el punto de corte de PSA y si alguna subpoblación de pacientes podría beneficiarse de alguna estrategia de rastreo como la propuestas ( $p$. ej. los más jóvenes o los que tienen antecedentes familiares de formas agresivas de CP).

Por lo tanto, e intentando ser consecuentes con nuestro deseo de no enfermar a nuestros pacientes con los efectos adversos de la medicina preventiva ${ }^{45}$, y por lo menos hasta que aparezca alguna eventual nueva evidencia a favor del rastreo poblacional de CP (lo que consideramos poco probable), recomendamos firmemente no implementarlo en población general asintomática, tal como acaba de expedirse la Fuerza de Tareas de Cuidados Preventivos de EE.UU.

Recibido el 05/ 06/12 y aceptado el 16/06/12.

Referencias

1. Screening for Prostate Cancer, Topic Page. U.S. Preventive Services Task Force. http://www.uspreventiveservicestaskforce.org/prostatecancerscreening.htm

2. Chou R, Croswell JM, Dana T, Bougatsos C, Blazina I, Fu R, et al. Screening for prostate cancer: a review of the evidence for the U.S. Preventive Services Task Force. Ann. Intern. Med. 2011 dic 6;155(11):762-71.

3. Schröder F y col. Screening and prostate-cancer mortality in a randomized European study. N. Engl. J. Med. 2009 Mar 26;360(13):1320-8.

tIttrtt Por otro lado, la proporción de individuos en el PLCO que eligió ser sometida a una conducta expectante por sobre un "tratamiento con intenciones curativas" o curativo fue levemente menor en el PLCO que en el ERSPC (10 vs. 19\%). Además, el PLCO evaluó un intervalo de rastreo más corto (anual vs. cada dos a siete años), lo que podría sugerir una tendencia de que el empleo de estrategias de rastreo menos intensivas y de tratamientos más conservadores podría ser más eficaz o menos dañino. Sin embargo no debemos dejar de considerar que estas aparentes discrepancias también fueron estadísticamente no significativas. 
4. Hugosson J, Carlsson S, Aus G, Bergdahl S, Khatami A, Lodding P, et al. Mortality results from the Göteborg randomised population-based prostate-cancer screening trial. Lancet Oncol. 2010 Ago;11(8):725-32.

5. Andriole GL, Crawford ED, Grubb RL 3rd, Buys SS, Chia D, Church TR, et al. Mortality results from a randomized prostate-cancer screening trial. N. Engl. J. Med. 2009 Mar 26;360(13):1310-9

6. Labrie F, Candas B, Cusan L, Gomez JL, Bélanger A, Brousseau G. et al. Screening decreases prostate cancer mortality: 11-year follow-up of the 1988 Quebec prospective randomized controlled trial.. Prostate. 2004;59311-8

7. Sandblom G, Varenhorst E, Lofman O, Rosell J, Carlsson P. Clinical consequences of screening for prostate cancer: 15 years follow-up of a randomised controlled trial in Sweden. Eur Urol. 2004;46717-23

8. U.S. Preventive Services Task Force. Screening for prostate cancer: U.S. Preventive Services Task Force recommendation statement. Ann Intern Med. $2008 ; 149: 185-91$. 9. Kjellman A, Akre O, Norming U, Törnblom M, Gustafsson O. 15-year followup of a population based prostate cancer screening study. J. Urol. 2009 Abr;181(4):1615-21; discussion 1621

10. Sandblom G, Varenhorst E, Rosell J, Löfman O, Carlsson P. Randomised prostate cancer screening trial: 20 year follow-up. BMJ. 2011;342:d1539

11. Kilpeläinen TP, Tammela TLJ, Määttänen L, Kujala P, Stenman U-H, Ala-Opas M, et al. False-positive screening results in the Finnish prostate cancer screening trial. Br. J. Cancer. 2010 Feb 2;102(3):469-74.

12. Croswell JM, Kramer BS, Kreimer AR, Prorok PC, Xu JL, Baker SG. et al. Cumulative incidence of false-positive results in repeated, multimodal cancer screening. Ann Fam Med. 2009;7212-22

13. Raaijmakers R, Kirkels WJ, Roobol MJ, Wildhagen MF, Schrder FH. Complication rates and risk factors of 5802 transrectal ultrasound-guided sextant biopsies of the prostate within a population-based screening program. Urology. 2002 Nov;60(5):826-30.

14. Bill-Axelson A, Holmberg L, Filén F, Ruutu M, Garmo H, Busch C, et al. Radical prostatectomy versus watchful waiting in localized prostate cancer: the Scandinavian prostate cancer group-4 randomized trial. J. Natl. Cancer Inst. 2008 Ago 20;100(16):1144-54.

15. Bill-Axelson A, Holmberg L, Ruutu M, Garmo H, Stark JR, Busch C, et al. Radical prostatectomy versus watchful waiting in early prostate cancer. N. Engl. J. Med. 2011 May 5;364(18):1708-17.

16. Bill-Axelson A, Holmberg L, Ruutu M, Häggman M, Andersson S-O, Bratell S, et al. Radical prostatectomy versus watchful waiting in early prostate cancer. N. Engl. J. Med. 2005 May 12;352(19):1977-84.

17. Holmberg L, Bill-Axelson A, Helgesen F, Salo JO, Folmerz P, Häggman M, et al. A randomized trial comparing radical prostatectomy with watchful waiting in early prostate cancer. N. Engl. J. Med. 2002 Sep 12;347(11):781-9.

18. Ladjevardi S, Sandblom G, Berglund A, Varenhorst E. Tumour grade, treatment, and relative survival in a population-based cohort of men with potentially curable prostate cancer. Eur. Urol. 2010 Abr;57(4):631-8.

19. Merglen A, Schmidlin F, Fioretta G, Verkooijen HM, Rapiti E, Zanetti R, et al. Short- and long-term mortality with localized prostate cancer. Arch. Intern. Med. 2007 Oct 8;167(18):1944-50.

20. Schymura MJ, Kahn AR, German RR, Hsieh M-C, Cress RD, Finch JL, et al. Factors associated with initial treatment and survival for clinically localized prostate cancer: results from the CDC-NPCR Patterns of Care Study (PoC1). BMC Cancer. 2010;10:152.

21. Stattin P, Holmberg E, Johansson J-E, Holmberg L, Adolfsson J, Hugosson J. Outcomes in localized prostate cancer: National Prostate Cancer Register of Sweden followup study. J. Natl. Cancer Inst. 2010 Jul 7;102(13):950-8.

22. Tewari A, Divine G, Chang P, Shemtov MM, Milowsky M, Nanus D, et al. Long-term survival in men with high grade prostate cancer: a comparison between conservative treatment, radiation therapy and radical prostatectomy--a propensity scoring approach. J. Urol. 2007 Mar;177(3):911-5.

23. Wong Y-N, Mitra N, Hudes G, Localio R, Schwartz JS, Wan F, et al. Survival associated with treatment vs observation of localized prostate cancer in elderly men. JAMA. 2006 Dic 13;296(22):2683-93.

24. Albertsen PC, Hanley JA, Penson DF, Barrows G, Fine J. 13-year outcomes following treatment for clinically localized prostate cancer in a population based cohort. J. Urol. 2007 Mar; 177(3):932-6.

25. Zhou EH, Ellis RJ, Cherullo E, Colussi V, Xu F, Chen W-D, et al. Radiotherapy and survival in prostate cancer patients: a population-based study. Int. J. Radiat. Oncol. Biol. Phys. 2009 Ene 1;73(1):15-23.

26. Johansson E, Bill-Axelson A, Holmberg L, Onelöv E, Johansson JE, Stenieck G; Scandinavian Prostate Cancer Group Study No 4. Time, symptom burden, androgen deprivation, and self-assessed quality of life after radical prostatectomy or watchful waiting: the Randomized Scandinavian Prostate Cancer Group Study No 4 (SPCG-4) clinical trial. Eur Urol. 2009;55;422-30.

27. Hoffman RM, Hunt WC, Gilliland FD, Stephenson RA, Potosky AL. Patient satisfaction with treatment decisions for clinically localized prostate carcinoma. Results from the Prostate Cancer Outcomes Study. Cancer. 2003;97:1653-62.

28. Litwin MS. Health-related quality of life after treatment for localized prostate cancer. Cancer. 1995;75:2000-3.

29. Schapira MM, Lawrence WF, Katz DA, McAuliffe TL, Nattinger AB. Effect of treatment on quality of life among men with clinically localized prostate cancer. Med Care. 2001;39:243-53.

30. Smith DP, King MT, Egger S, Berry MP, Stricker PD, Cozzi P, et al. Quality of life three years after diagnosis of localised prostate cancer: population based cohort study. BMJ. 2009;339:b4817.

31. Siegel T, Moul JW, Spevak M, Alvord WG, Costabile RA. The development of erectile dysfunction in men treated for prostate cancer. J Urol. 2001;165:430-5.

32. Bacon CG, Giovannucci E, Testa M, Kawachi I. The impact of cancer treatment on quality of life outcomes for patients with localized prostate cancer. J Urol. 2001;166:1804-10.

33. Galbraith ME, Ramirez JM, Pedro LW. Quality of life, health outcomes, and identity for patients with prostate cancer in five different treatment groups. Oncol Nurs Forum. 2001;28:551-60.

34. Litwin MS, Hays RD, Fink A, Ganz PA, Leake B, Leach GE, et al. Quality-of-life outcomes in men treated for localized prostate cancer. JAMA. 1995;273:129-35.

35. Litwin MS, Lubeck DP, Spitalny GM, Henning JM, Carroll PR. Mental health in men treated for early stage prostate carcinoma: a posttreatment, longitudinal quality of life analysis from the Cancer of the Prostate Strategic Urologic Research Endeavor. Cancer. 2002;95:54-60.

36. Lubeck DP, Litwin MS, Henning JM, Stoddard ML, Flanders SC, Carroll PR. Changes in health-related quality of life in the first year after treatment for prostate cancer: results from CaPSURE. Urology.

37. Smith DS, Carvalhal GF, Schneider K, Krygiel J, Yan Y, Catalona WJ. Quality-of-life outcomes for men with prostate carcinoma detected by screening. Cancer. 2000;88:1454-63.

38. Ware JE Jr, Snow KK, Kosinski M, Gandek B. SF-36 health survey manual and interpretation guide. Boston,: Health Institute, New England Medical Centre, 1993.

39. Yao SL, Lu-Yao G. Population-based study of relationships between hospital volume of prostatectomies, patient outcomes, and length of hospital stay. J Natl Cancer Inst. 1999;91:1950-6.

40. Steineck G, Helgesen F, Adolfsson J, Dickman PW, Johansson JE, Norlén BJ, et al; Scandinavian Prostatic Cancer Group Study Number 4. Quality of life after radical prostatectomy or watchful waiting. N Engl J Med. 2002;347:790-6.

41. Fransson P, Damber JE, Tomic R, Modig H, Nyberg G, Widmark A. Quality of life and symptoms in a randomized trial of radiotherapy versus deferred treatment of localized prostate carcinoma. Cancer. 2001;92:3111-9.

42. Thong MS, Mols F, Kil PJ, Korfage IJ, van de Poll-Franse LV. Prostate cancer survivors who would be eligible for active surveillance but were either treated with radiotherapy or managed expectantly: comparisons on long-term quality of life and symptom burden. BJU Int. 2010;105:652-8.

43. Fransson P, Damber JE, Widmark A. Health-related quality of life 10 years after external beam radiotherapy or watchful waiting in patients with localized prostate cancer. Scand J Urol Nephrol. 2009;43:119-26.

44. Talcott JA, Manola J, Clark JA, Kaplan I, Beard CJ, Mitchell SP, et al. Time course and predictors of symptoms after primary prostate cancer therapy. J Clin Oncol. 2003;21:3979-86.

45. Rubinstein E. Los nuevos enfermos: Ventajas y desventajas de la medicina preventiva. Buenos Aires: Delhospital Ediciones; 2012. 